\title{
Psychiatric Nurses Providing Post-Stroke Depression Education to Care Partners of Stroke: A Pilot Study
}

PATRICIA ZULIM, BS, RN, PMHN-BC https://orcid.org/0000-0001-9543-7966

SONJA E. STUTZMAN, PhD https://orcid.org/0000-0002-8902-2067

\author{
ANJALI PERERA, BSN \\ https://orcid.org/0000-0001-5530-3445
}

DAIWAI M. OLSON, PhD, RN, CCRN, FNCS

https://orcid.org/0000-0002-9280-078X

University of Texas Southwestern Medical Center, Dallas, Texas, USA
Corresponding author's email: DaiWai.OIson@UTSouthwestern.edu

Abstract

Background: Stroke survivors are a higher risk for post-stroke depression (PSD) after they return home from the hospital. Given that many stroke patients have friends or family members (care partners) present after hospital discharge, this pilot study examined the utility of psychiatric nurses educating family members on how to recognize PSD.

Methods: This pilot study identified stroke patients and their care partner while they were in the hospital, to provide education on depressive symptoms, community resources, safety resources (e.g., suicide hotline), how to address symptoms of depression with their care partner. A descriptive observational design was used wherein a psychiatric delivered PSD education to the patient's care partner (instead of the patient). Knowledge of depression was assessed immediately prior to-, immediately after-, and at a 2-week follow-up after-, the education intervention.

Results: This pilot study was not powered to detect a statistically significant difference. However, among the 20 subjects (mean age 61 years), fewer scores fell in the lower range (less knowledge of depression) immediately after the education and at the 2-week follow-up phone call.

Conclusion: This study demonstrates the potential of multi-disciplinary nursing consultation to improve outcomes.

Keywords: care-partner, care partner depression, nursing, stroke 


\section{Introduction}

ealth education has repeatedly been found to be a predictor to improving knowledge of stroke signs and symptoms (Lennon, Blake, Booth, Pollock, \& Lawrence, 2018). Stroke knowledge

has been linked to improved outcomes and reduced delay in treatment seeking behavior (Pandian et al., 2018). It stands to reason that a nurse who specializes in stroke is more likely to provide high quality stroke education than a nurse who specializes in another field. Similarly, nurses who specialize in psychiatric illness (including depression) are more prepared to provide depressionscreening education than nurses who specialize in stroke. Self-recognition of depression is poorly studied with estimates ranging from $21 \%$ to $62 \%$ (Caplan \& Buyske, 2015). This suggests that those with depression may be less likely than others to recognize their condition. Stroke is a known risk factor for depression and neuroscience nurses are often tasked with screening for depression, and to educate patients about, post stroke depression (PSD) (Cai, Stewart, Mueller, Li, \& Shen, 2018; Trotter, Denny, \& Evanson, 2019). Noting the aforementioned limitations, the purpose of this pilot study was to explore the efficacy of PSD education administered to care partners by a psychiatric nurse.

\section{Background}

Stroke is the leading cause of long-term disability in the United States (Thom et al., 2006). As the population is living longer, the number of patients experiencing stroke is increasing (Benjamin et al., 2017). On average, a stroke occurs every 45 seconds, with women having higher incidence than men. Nurses working with patients who have had a stroke are oftentimes responsible for answering patient and care partner or caregiver questions regarding the recovery process posthospital discharge. Additionally, these nurses are expected to provide post-discharge education on a number of topics (e.g., medications, transition planning, ADL training, secondary stroke injury awareness, new stroke symptom awareness, etc.). This education is usually provided to the patients, and if a care partner is available at the time of the education, they can also listen in. The amount of information provided to the stroke survivor can be overwhelming and adding additional information, such as potentially trying to recognize depression symptoms, can add to confusion or being overwhelmed. Stroke nurses are trained on depressive symptoms and stroke recovery, but oftentimes these are not discussed in tandem. There is a need for patient and family PSD education, which is included as part of the Stroke Certified Registered Nurse Training.

Stroke survivors have a high rate of injury that requires care beyond the hospital that is provided by a family member, care partner, or employed health aid, with the majority of the burden falling on those that are closest to the stroke survivor (Olson, 2017). A care partner is distinguished from a member, caregiver, or health aid as the mutually benefiting dyad (Lu, Martensson, Zhao, \& Johansson, 2019). The dyad promotes common goals that supports interdependence. This is a 
divergence from the traditional caregiver holding the majority of the burden to support the receiver (Bennett, Wang, Moore, \& Nagle, 2017). Emerging evidence supports that care partners also play a vital role in the stroke patient's recovery (Olson et al., 2011). The care partner involvement in the discharge education is usually optional and based on availability, therefore the information can be provided second hand to the care partner or not at all.

\section{Depression and Stroke}

Depression can be characterized as acute or chronic. Acute depression has a high recovery rate, and usually occurs after a traumatic incident. Chronic depression is defined as depression that has lasted for more than two years, and has a low recovery rate (Keller \& Shapiro, 1982). Major depressive disorder (MDD) is a type of chronic depression defined by the American Psychiatric Association as those cases showing persistent signs of anhedonia, hopelessness, helplessness, over sleeping, and more. To be diagnosed with MDD, patients have to exhibit at least five of these symptoms for two weeks.

Full-time care of a stroke survivor requires dedication that can be overwhelming and can take a toll on the care partner (Lu et al., 2019). Depression, stroke, and disabilities have been studied in the literature and been shown to overlap presentation of symptoms and side effects (El Husseini et al., 2017). Stroke survivors and their care partners have an elevated risk of developing depression. Researchers have reported that providing care partners with support can decrease depressive symptoms (Berg, Palomaki, Lonnqvist, Lehtihalmes, \& Kaste, 2005; National Stroke Association, 2018).

Studies suggest that risk assessment tools are useful to aid clinicians and patients in determining potential hazards that can cause stroke and related disabilities such as depression (Berg, Lonnqvist, Palomaki, \& Kaste, 2009; Goldstein, et al., 2006). Depression after stroke may result or manifest as physical disabilities, and may ultimately incapacitate stroke survivors (Towfighi et al., 2017). These symptoms may be very difficult for the stroke survivor to recognize, therefore a partner specific education of symptoms of depression may be helpful. Approximately $7 \%$ of adults in the United States are diagnosed with depression every year.

Statistics on help seeking amongst those that are depressed vary and are based on socioeconomic status, age, social support, and culture. For stroke survivors, research has shown that there may be a link between stroke, depression, delayed help seeking, and poorer long term prognosis (Goodwin \& Devanand, 2008). Even though stroke survivors are at higher risk for depression there has been little research done on the help-seeking for depression in this population.

Stroke recovery symptoms and depressive symptoms can co-exist (Kapoor et al., 2019). Acute depression can present in patients and care partners after a stroke. Research findings suggest an increase in the incidence of depression among care partners of stroke patients. In particular, one 
study showed that although there was no increase in depression among patients and care partners within three months after stroke, there was a significant increase of depression symptoms among caretakers at twelve months, who did not have any support programs (Kotila, Numminen, Waltimo, \& Kaste, 1998). Another study suggests that younger stroke patients have higher mortality and that there is a positive association between post-stroke depression and mortality (Ayerbe, Ayis, Crichton, Rudd, \& Wolfe, 2014). These statistics have led to education of stroke survivors and their care partners prior to discharge, yet many times this education is provided amongst an overwhelming amount of other information (medications, physical therapy schedules, follow-up visits, and other discharge information). The information provided about recognition of depressive symptoms can be easily forgotten by the patient or care partner or not addressed by the provider.

\section{Methods}

This pilot study used a descriptive observational design to evaluate the efficacy of targeted depression symptom recognition education for care partners of stroke survivors. This study fills a gap in the literature by evaluating two novel approaches to post-stroke education: 1) the population being studied is the care partner, and 2) the intervention is an educational intervention delivered by a psychiatric nurse with expertise in depression and psychiatric nursing. Prior to any study procedures, approval was authorized by the Institutional Review Board (ethical review) and consent was obtained from all participants. Patients were not enrolled in the study and all patients continued to receive the standard of care including PSD education.

The intervention was teaching care partners to recognize PSD symptoms in patients who have had a stroke. The PSD education was designed by the psychiatric nurse, who, along with one other member of the care team, a Ph.D. prepared researcher with experience in psychiatric measures), provided education to care partners. The study team provided the care partners with targeted education of depressive symptoms and then evaluated the care partner confidence and knowledge to recognize depressive symptoms 2-weeks post-discharge. The education consisted of training on changing thoughts, emotions, physical, and behavioral symptoms of depression that the care partner may see upon discharge. Additional depression resources, suicide help lines, and community resources were provided to the care partner about the education given. All resources were publically available information that were written in layman's terms to be easily understood. The outcome of interest for this study is knowledge of signs and symptoms of depression.

Eligible participants were care partners of stroke patients admitted to the enrolling hospital if the care partner and the patient were at least 40 years of age. Participants were verbally consented and participated in the education module while the stroke patient was still in the hospital. The recruitment phase of the study lasted seven months. Before and after the education session, the Knowledge of Later Life Depression Scale Modified (KLLDS) test was administered to assess the participant's knowledge and confidence in recognizing depression symptoms (Karantzas, Davison, 


\section{JOURNAL of HEALTH and CARING SCIENCES}

McCabe, Mellor, \& Beaton, 2012). The scale is 10 items that test the knowledge of recognition of depressive symptoms, specifically in the elderly patient. Responses range from strongly disagree to strongly agree. The scale has shown adequate internal consistency and internal reliability $(\alpha=0.82)$ (Karantzas et al., 2012). Seven additional items, with responses ranging from strongly disagree to strongly agree, were included to address care partner knowledge of PSD. The surveys were administered three times throughout the study: baseline (prior to education), post-test (immediately following education), and follow-up (2-week follow-up via phone).

\section{Results}

Of the 23 care partners enrolled in the study, three were excluded for not meeting the age eligibility requirements. The study subjects $(n=20)$ mean age was $60.6(S D=12.5)$ years; $13(65 \%)$ of the care partners were female and $10(50 \%)$ were Caucasian (Table 1). The mean age for patients to whom care partners were related was $64.9(\mathrm{SD}=12.6)$ years. All 20 care partners completed the pre-assessment and participated in the educational intervention. There were 6 subjects lost to followup for whom post-intervention scores are not available.

Table 1. The Demographic characteristics of the care partners.

\begin{tabular}{|c|c|}
\hline Variable & Mean (SD) \\
\hline \multirow[t]{2}{*}{ Age (years) } & $56.4(16.4)$ \\
\hline & $N(\%)$ \\
\hline \multicolumn{2}{|l|}{ Relationship to patient } \\
\hline Married & 15 \\
\hline Cohabitating & $(68.2 \%) 3$ \\
\hline Live separately & $(13.6 \%)$ \\
\hline Sex & 15 \\
\hline Female & $(65.2 \%) 8$ \\
\hline Male & $(34.8 \%)$ \\
\hline \multicolumn{2}{|l|}{ Race } \\
\hline Caucasian & $11(47.8 \%)$ \\
\hline African & $3(13.05 \%)$ \\
\hline American & $3(13.05 \%)$ \\
\hline Asian Other/not given & $6(26.1 \%)$ \\
\hline \multicolumn{2}{|l|}{ Ethnicity } \\
\hline Hispanic & $5(21.7 \%)$ \\
\hline Not Hispanic & $17(74.0 \%)$ \\
\hline Not given & $1(4.3 \%)$ \\
\hline
\end{tabular}

The SAS PROC GLM for ANOVA was used to explore the omnibus test of difference in mean scores. Although the KLLDS scores increased over time, there was no difference in average KLLDS scores measured at the three time points: pre-test $=6.25(1.25)$; post-test $=6.28(1.36)$; and follow-up $=6.71(0.99) ; p=0.5065$. The range of KLLDS scores increased slightly between from pre- 
test $(3.0,8.0)$, post-test $(5.0,7.0)$, and follow-up $(5.0,8.0)$. Scores for the additional 7 items increased between pre-test $=3.45(1.05)$ and post-test $=3.67(0.84)$, but decreased at follow-up $=3.14(0.86)$, with no statistically significant differences $(p=0.2978)$. The scores ranged from $1-4$ pre-test, from 3 to 4 post-test, and from 2 to 4 at 2-week follow-up.

\section{Discussion}

This pilot demonstrates that care partner education is feasible and that a psychiatric nurse is able to readily provide education on depressive symptoms. Given that stroke patients may be discharged with varied levels of independence, targeting education to care partners may reduce patient stress (Pucciarelli et al., 2019). While the study was not powered to detect a statistically significant difference in pre-test versus post-test scores, the available data suggests that care partners did benefit from the educational intervention.

Looking for a scale that would fit our study on accessing care partner's knowledge on recognizing signs and symptoms of depression on post stroke patients was challenging. Most PSD education is targeted to educate the patient. To our knowledge, the KLLDS has not been tested to assess stroke care partner depression knowledge. During the process we found that KLLDS, which it was not specific to the post-stroke depression study, was able access some of the care partner's knowledge about depression, yet it fell short of capturing the overlap of stroke and depressive symptoms. The efficacy of the KLLDS administration over the phone was not found in the literature and may need further inquiry.

There was a trend towards older adults demonstrating a better understanding of the symptomology of depression than younger care partners. It is possible that the older care partners have already received education on depression in later life and had a stronger baseline knowledge of the symptoms. This suggests that younger care partners of stroke survivors may need additional education as they are not as in tune to the symptoms associated with stroke and depression.

Education of overlap of post-stroke and depressive symptoms is important. Education is usually provided by the nurse assigned to care for the patient. It may make sense for this education to be provided by someone with training in psychiatric disorders and depression symptom recognition. In modern nursing, no nurse can truly be a specialist in every discipline. The concept of nurses from one specialty collaborating with nurses from another specialty is not new. Moreover, physicians frequently consult specialty services from other physicians. However, it is novel for nurses with neuroscience training to consult and delegate education to nurses with psychiatric training. Future research is needed to compare nurses and care partners' knowledge of post-stroke depression to truly understand if a nurse specializing in psychiatric disorders is needed to provide this type of education. 


\section{JOURNAL of HEALTH and CARING SCIENCES}

\section{Limitations}

The limitation of this pilot study included $30 \%$ lost to follow-up. This may have been due to the close time parameter around the follow up phone call, as two weeks after the patient discharges can still be a fairly hectic time for care partners. Additionally, the sample size of this pilot study is relatively small, and therefore the generalizability of the results is limited. The absence of a scale that has been validated to understand knowledge of post-stroke depression knowledge in care partners is a major limitation. Additional items were added to the pre-test and post-test and scored independently from the KLLDS. Additional scale development is needed to evaluate PSD knowledge in care partners.

\section{Conclusion}

To our knowledge this is the first study to demonstrate a benefit to PSD education delivered by psychiatric nurses focusing on the educational needs of care partners. This study demonstrates the potential for nurses to collaborate to provide education to care partners and also that education with a two week follow up maybe efficacious in showing differences in knowledge. Future research is needed to determine how to best educate and evaluate care partners of stroke on depressive symptoms.

\section{Funding}

This research did not receive any specific grant from funding agencies in the public, commercial or not-for-profit sectors.

\section{References}

Ayerbe, L., Ayis, S., Crichton, S. L., Rudd, A. G., \& Wolfe, C. D. (2014). Explanatory factors for the increased mortality of stroke patients with depression. Neurology, 83(22), 2007-2012. doi:10.1212/WNL.0000000000001029

Benjamin, E. J., Blaha, M. J., Chiuve, S. E., Cushman, M., Das, S. R., Deo, R., . . Muntner, P. (2017). Heart Disease and Stroke Statistics-2017 Update: A Report From the American Heart Association. Circulation, 135(10), e146-e603. doi:10.1161/cir.0000000000000485

Bennett, P. N., Wang, W., Moore, M., \& Nagle, C. (2017). Care partner: A concept analysis. Nursing Outlook, 65(2), 184-194. doi:10.1016/j.outlook.2016.11.005

Berg, A., Lonnqvist, J., Palomaki, H., \& Kaste, M. (2009). Assessment of depression after stroke: a comparison of different screening instruments. Stroke, 40(2), 523-529. doi:10.1161/strokeaha.108.527705 
Berg, A., Palomaki, H., Lonnqvist, J., Lehtihalmes, M., \& Kaste, M. (2005). Depression among caregivers of stroke survivors. Stroke, 36(3), 639-643. doi:10.1161/01.STR.0000155690.04697.c0

Cai, W., Stewart, R., Mueller, C., Li, Y.-J., \& Shen, W.-D. (2018). Poststroke depression and risk of stroke recurrence and mortality: protocol of a meta-analysis and systematic review. BMJ Open, 8(12), e026316-e026316. doi:10.1136/bmjopen-2018-026316

Caplan, S., \& Buyske, S. (2015). Depression, Help-Seeking and Self-Recognition of Depression among Dominican, Ecuadorian and Colombian Immigrant Primary Care Patients in the Northeastern United States. International Journal of Environmental Research and Public Health, 12(9), 10450-10474. doi:10.3390/ijerph120910450

El Husseini, N., Goldstein, L. B., Peterson, E. D., Zhao, X., Olson, D. M., Williams, J. W., Jr., . . Laskowitz, D. T. (2017). Depression Status Is Associated with Functional Decline Over 1-Year Following Acute Stroke. Journal of Stroke and Cerebrovascular Diseases, 26(7), 1393-1399. doi:10.1016/j.jstrokecerebrovasdis.2017.03.026

Goldstein, L. B., Adams, R., Alberts, M. J., Appel, L. J., Brass, L. M., Bushnell, C. D., . . American Academy of, N. (2006). Primary prevention of ischemic stroke: a guideline from the American Heart Association/American Stroke Association Stroke Council: cosponsored by the Atherosclerotic Peripheral Vascular Disease Interdisciplinary Working Group; Cardiovascular Nursing Council; Clinical Cardiology Council; Nutrition, Physical Activity, and Metabolism Council; and the Quality of Care and Outcomes Research Interdisciplinary Working Group: the American Academy of Neurology affirms the value of this guideline. Stroke, 37(6), 1583-1633. doi:10.1161/01.STR.0000223048.70103.F1

Goodwin, R. D., \& Devanand, D. P. (2008). Stroke, depression, and functional health outcomes among adults in the community. Journal of Geriatric Psychiatry and Neurology, 21(1), 41-46.

Kapoor, A., Lanctot, K. L., Bayley, M., Herrmann, N., Murray, B. J., \& Swartz, R. H. (2019). Screening for PostStroke Depression and Cognitive Impairment at Baseline Predicts Long-Term Patient-Centered Outcomes After Stroke. Journal of Geriatric Psychiatry and Neurology, 32(1), 40-48. doi:10.1177/0891988718819859

Karantzas, G. C., Davison, T. E., McCabe, M. P., Mellor, D., \& Beaton, P. (2012). Measuring carers' knowledge of depression in aged care settings: The Knowledge of Late Life Depression Scale-Revised. Journal of Affective Disorders, 138(3), 417-424.

Keller, M. B., \& Shapiro, R. W. (1982). "Double depression": superimposition of acute depressive episodes on chronic depressive disorders. The American Journal of Psychiatry, 139(4), 438-442. doi:10.1176/ajp.139.4.438

Kotila, M., Numminen, H., Waltimo, O., \& Kaste, M. (1998). Depression after stroke: results of the FINNSTROKE Study. Stroke, 29(2), 368-372.

Retrieved from https://www.ncbi.nlm.nih.gov/pubmed/9472876

Lennon, O., Blake, C., Booth, J., Pollock, A., \& Lawrence, M. (2018). Interventions for behaviour change and self-management in stroke secondary prevention: protocol for an overview of reviews. Systematic Reviews, 7(1), 231. doi:10.1186/s13643-018-0888-1

Lu, Q., Martensson, J., Zhao, Y., \& Johansson, L. (2019). Living on the edge: Family caregivers' experiences of caring for post-stroke family members in China: A qualitative study. International Journal of Nursing Studies, 94, 1-8. doi:10.1016/j.jinurstu.2019.02.016

National Stroke Association. (2018). caregivers at risk for depression. Retrieved from http://www.stroke.org/news-release/caregivers-risk-depression 


\section{JOURNAL of HEALTH and CARING SCIENCES}

Olson, D. M. (2017). Caregiver or Care-partner. Journal of Neuroscience Nursing, 49(3), 136. doi:10.1097/jnn.0000000000000288

Olson, D. M., Prvu-Bettger, J., Alexander, K. P., Kendrick, A. S., Irvine, J. R., Wing, L., . . Graffagnino, C. (2011). Transition of Care for Acute Stroke and Myocardial Infarction Patients: From Hospitalization to Rehabilitation, Recovery, and Secondary Prevention. Evidence Report No. 202. (Prepared by the Duke Evidence-based Practice Center under Contract No. 290-2007-10066-I.) Agency for Healthcare Research and Quality AHRQ Publication No. 11(2)-E011.

Pandian, J. D., Gall, S. L., Kate, M. P., Silva, G. S., Akinyemi, R. O., Ovbiagele, B. I., . . Thrift, A. G. (2018). Prevention of stroke: a global perspective. Lancet, 392(10154), 1269-1278. doi:10.1016/s0140-6736(18)31269-8

Pucciarelli, G., Ausili, D., Rebora, P., Arisido, M. W., Simeone, S., Alvaro, R., \& Vellone, E. (2019). Formal and informal care after stroke: A longitudinal analysis of survivors' post rehabilitation hospital discharge. Journal of Advanced Nursing. doi:10.1111/jan.13998

Thom, T., Haase, N., Rosamond, W., Howard, V. J., Rumsfeld, J., Manolio, T., . . Stroke Statistics, S. (2006). Heart disease and stroke statistics--2006 update: a report from the American Heart Association Statistics Committee and Stroke Statistics Subcommittee. Circulation, 113(6), e85-151. doi:10.1161/CIRCULATIONAHA.105.171600

Towfighi, A., Ovbiagele, B., El Husseini, N., Hackett, M. L., Jorge, R. E., Kissela, B. M., . . Williams, L. S. (2017). Poststroke Depression: A Scientific Statement for Healthcare Professionals From the American Heart Association/American Stroke Association. Stroke, 48(2), e30-e43. doi:10.1161/str.0000000000000113

Trotter, T. L., Denny, D. L., \& Evanson, T. A. (2019). Reliability and validity of the Patient Health Questionaire9 as a screening tool for poststroke depression. Journal of Neuroscience Nursing, 51(4), 6.

\section{About the Authors}

Patricia Zulim, BS, RN, PMHN-BC, is a Mental Health Staff Nurse at the UT Southwestern Medical Center in Dallas TX USA

Anjali Perera, BSN, is a Neurocritical Care Staff Nurse at the UT Southwestern Medical Center in Dallas TX USA

Sonja E. Stutzman, PhD, is the Program Manager for the Neuroscience Nursing Research Center at the University of Texas Southwestern Medical Center in Dallas, TX USA

Daiwai M. Olson, PhD, RN, CCRN, FNCS, is a Professor of Neurology University of Texas Southwestern and a Neurocritical Care Nurse at the UT Southwestern Medical Center in Dallas TX USA. 